\title{
Positive Solutions of Singular Multipoint Boundary Value Problems for Systems of Nonlinear Second-Order Differential Equations on Infinite Intervals in Banach Spaces
}

\author{
Xingqiu Zhang \\ School of Mathematics, Liaocheng University, Liaocheng, Shandong 252059, China \\ Correspondence should be addressed to Xingqiu Zhang, zhxq197508@163.com \\ Received 27 April 2009; Accepted 12 June 2009 \\ Recommended by Donal O’Regan
}

The cone theory together with Mönch fixed point theorem and a monotone iterative technique is used to investigate the positive solutions for some boundary problems for systems of nonlinear second-order differential equations with multipoint boundary value conditions on infinite intervals in Banach spaces. The conditions for the existence of positive solutions are established. In addition, an explicit iterative approximation of the solution for the boundary value problem is also derived.

Copyright (c) 2009 Xingqiu Zhang. This is an open access article distributed under the Creative Commons Attribution License, which permits unrestricted use, distribution, and reproduction in any medium, provided the original work is properly cited.

\section{Introduction}

In recent years, the theory of ordinary differential equations in Banach space has become a new important branch of investigation (see, e.g., [1-4] and references therein). By employing a fixed point theorem due to Sadovskii, Liu [5] investigated the existence of solutions for the following second-order two-point boundary value problems (BVP for short) on infinite intervals in a Banach space $E$ :

$$
\begin{gathered}
x^{\prime \prime}(t)=f\left(t, x(t), x^{\prime}(t)\right), \quad t \in J, \\
x(0)=x_{0}, \quad x^{\prime}(\infty)=y_{\infty},
\end{gathered}
$$

where $f \in C[J \times E \times E, E], J=[0,+\infty), x^{\prime}(\infty)=\lim _{t \rightarrow \infty} x^{\prime}(t)$. On the other hand, the multipoint boundary value problems arising naturally from applied mathematics and physics have been 
studied so extensively in scalar case that there are many excellent results about the existence of positive solutions (see, i.e., [6-12] and references therein). However, to the best of our knowledge, only a few authors $[5,13,14]$ have studied multipoint boundary value problems in Banach spaces and results for systems of second-order differential equation are rarely seen. Motivated by above papers, we consider the following singular $m$-point boundary value problem on an infinite interval in a Banach space $E$

$$
\begin{gathered}
x^{\prime \prime}(t)+f\left(t, x(t), x^{\prime}(t), y(t), y^{\prime}(t)\right)=0, \\
y^{\prime \prime}(t)+g\left(t, x(t), x^{\prime}(t), y(t), y^{\prime}(t)\right)=0, \quad t \in J_{+}, \\
x(0)=\sum_{i=1}^{m-2} \alpha_{i} x\left(\xi_{i}\right), \quad x^{\prime}(\infty)=x_{\infty}, \\
y(0)=\sum_{i=1}^{m-2} \beta_{i} y\left(\xi_{i}\right), \quad y^{\prime}(\infty)=y_{\infty},
\end{gathered}
$$

where $J=[0, \infty), J_{+}=(0, \infty), \alpha_{i}, \beta_{i} \in[0,+\infty)$ and $\xi_{i} \in(0,+\infty)$ with $0<\xi_{1}<\xi_{2}<\cdots<$ $\xi_{m-2}<+\infty, 0<\sum_{i=1}^{m-2} \alpha_{i}<1,0<\sum_{i=1}^{m-2} \beta_{i}<1, \sum_{i=1}^{m-2} \alpha_{i} \xi_{i} /\left(1-\sum_{i=1}^{m-2} \alpha_{i}\right)>1, \sum_{i=1}^{m-2} \beta_{i} \xi_{i} /(1-$ $\left.\sum_{i=1}^{m-2} \beta_{i}\right)>1$. In this paper, nonlinear terms $f$ and $g$ may be singular at $t=0, x, y=\theta$, and/or $x^{\prime}, y^{\prime}=\theta$, where $\theta$ denotes the zero element of Banach space $E$. By singularity, we mean that $\left\|f\left(t, x_{0}, x_{1}, y_{0}, y_{1}\right)\right\| \rightarrow \infty$ as $t \rightarrow 0^{+}$or $x_{i}, y_{i} \rightarrow \theta(i=0,1)$.

Very recently, by using Shauder fixed point theorem, Guo [15] obtained the existence of positive solutions for a class of $n$ th-order nonlinear impulsive singular integro-differential equations in a Banach space. Motivated by Guo's work, in this paper, we will use the cone theory and the Mönch fixed point theorem combined with a monotone iterative technique to investigate the positive solutions of BVP (1.2). The main features of the present paper are as follows. Firstly, compared with [5], the problem we discussed here is systems of multipoint boundary value problem and nonlinear term permits singularity not only at $t=0$ but also at $x, y, x^{\prime}, y^{\prime}=\theta$. Secondly, compared with [15], the relative compact conditions we used are weaker. Furthermore, an iterative sequence for the solution under some normal type conditions is established which makes it very important and convenient in applications.

The rest of the paper is organized as follows. In Section 2, we give some preliminaries and establish several lemmas. The main theorems are formulated and proved in Section 3. Then, in Section 4, an example is worked out to illustrate the main results.

\section{Preliminaries and Several Lemmas}

Let

$$
\begin{gathered}
F C[J, E]=\left\{x \in C[J, E]: \sup _{t \in J} \frac{\|x(t)\|}{t+1}<\infty\right\}, \\
D C^{1}[J, E]=\left\{x \in C^{1}[J, E]: \sup _{t \in J} \frac{\|x(t)\|}{t+1}<\infty, \sup _{t \in J}\left\|x^{\prime}(t)\right\|<\infty\right\} .
\end{gathered}
$$


Evidently, $C^{1}[J, E] \subset C[J, E], D C^{1}[J, E] \subset F C[J, E]$. It is easy to see that $F C[J, E]$ is a Banach space with norm

$$
\|x\|_{F}=\sup _{t \in J} \frac{\|x(t)\|}{t+1}
$$

and $D C^{1}[J, E]$ is also a Banach space with norm

$$
\|x\|_{D}=\max \left\{\|x\|_{F},\left\|x^{\prime}\right\|_{C}\right\}
$$

where

$$
\left\|x^{\prime}\right\|_{C}=\sup _{t \in J}\left\|x^{\prime}(t)\right\|
$$

Let $X=D C^{1}[J, E] \times D C^{1}[J, E]$ with norm

$$
\|(x, y)\|_{X}=\max \left\{\|x\|_{D},\|y\|_{D}\right\}, \quad \forall(x, y) \in X .
$$

Then $\left(X,\|\cdot, \cdot\|_{X}\right)$ is also a Banach space. The basic space using in this paper is $\left(X,\|\cdot, \cdot\|_{X}\right)$.

Let $P$ be a normal cone in $E$ with normal constant $N$ which defines a partial ordering in $E$ by $x \leq y$. If $x \leq y$ and $x \neq y$, we write $x<y$. Let $P_{+}=P \backslash\{\theta\}$. So, $x \in P_{+}$if and only if $x>\theta$. For details on cone theory, see [4].

In what follows, we always assume that $x_{\infty} \geq x_{0}^{*}, y_{\infty} \geq y_{0}^{*}, x_{0}^{*}, y_{0}^{*} \in P_{+}$. Let $P_{0 \lambda}=\{x \in$ $\left.P: x \geq \lambda x_{0}^{*}\right\}, P_{1 \lambda}=\left\{y \in P: y \geq \lambda y_{0}^{*}\right\}(\lambda>0)$. Obviously, $P_{0 \lambda}, P_{1 \lambda} \subset P_{+}$for any $\lambda>0$. When $\lambda=1$, we write $P_{0}=P_{01}, P_{1}=P_{11}$, that is, $P_{0}=\left\{x \in P: x \geq x_{0}^{*}\right\}, P_{1}=\left\{y \in P: y \geq y_{0}^{*}\right\}$. Let $P(F)=\{x \in F C[J, E]: x(t) \geq \theta, \forall t \in J\}$, and $P(D)=\left\{x \in D C^{1}[J, E]: x(t) \geq \theta, x^{\prime}(t) \geq\right.$ $\theta, \forall t \in J\}$. It is clear, $P(F), P(D)$ are cones in $F C[J, E]$ and $D C^{1}[J, E]$, respectively. A map $(x, y) \in D C^{1}[J, E] \cap C^{2}\left[J_{+}^{\prime}, E\right]$ is called a positive solution of BVP $(1.2)$ if $(x, y) \in P(D) \times P(D)$ and $(x(t), y(t))$ satisfies (1.2).

Let $\alpha, \alpha_{F}, \alpha_{D}, \alpha_{X}$ denote the Kuratowski measure of noncompactness in $E, F C[J, E], D C^{1}[J, E]$ and $X$, respectively. For details on the definition and properties of the measure of noncompactness, the reader is referred to [1-4]. Let $L\left[J_{+}, J\right]$ be all Lebesgue measurable functions from $J_{+}$to $J$. Denote

$$
D_{0}=\frac{1}{1-\sum_{i=1}^{m-2} \alpha_{i}} \sum_{i=1}^{m-2} \alpha_{i} \xi_{i}, \quad D_{1}=\frac{1}{1-\sum_{i=1}^{m-2} \beta_{i}} \sum_{i=1}^{m-2} \beta_{i} \xi_{i}
$$


Let us list some conditions for convenience.

$\left(\mathrm{H}_{1}\right) f, g \in C\left[J_{+} \times P_{0 \lambda} \times P_{0 \lambda} \times P_{1 \lambda} \times P_{1 \lambda}, P\right]$ for any $\lambda>0$ and there exist $a_{i}, b_{i}, c_{i} \in L\left[J_{+}, J\right]$ and $h_{i} \in C\left[J_{+} \times J_{+}, J\right](i=0,1)$ such that

$$
\begin{gathered}
\left\|f\left(t, x_{0}, x_{1}, y_{0}, y_{1}\right)\right\| \leq a_{0}(t)+b_{0}(t) h_{0}\left(\left\|x_{0}\right\|,\left\|x_{1}\right\|,\left\|y_{0}\right\|,\left\|y_{1}\right\|\right), \\
\forall t \in J_{+}, x_{i} \in P_{0}, y_{i} \in P_{1}(i=0,1), \\
\left\|g\left(t, x_{0}, x_{1}, y_{0}, y_{1}\right)\right\| \leq a_{1}(t)+b_{1}(t) h_{1}\left(\left\|x_{0}\right\|,\left\|x_{1}\right\|,\left\|y_{0}\right\|,\left\|y_{1}\right\|\right), \\
\forall t \in J_{+}, x_{i} \in P_{0}, y_{i} \in P_{1}(i=0,1), \\
\frac{\left\|f\left(t, x_{0}, x_{1}, y_{0}, y_{1}\right)\right\|}{c_{0}(t)\left(\left\|x_{0}\right\|+\left\|x_{1}\right\|+\left\|y_{0}\right\|+\left\|y_{1}\right\|\right)} \longrightarrow 0, \quad \frac{\left\|g\left(t, x_{0}, x_{1}, y_{0}, y_{1}\right)\right\|}{c_{1}(t)\left(\left\|x_{0}\right\|+\left\|x_{1}\right\|+\left\|y_{0}\right\|+\left\|y_{1}\right\|\right)} \longrightarrow 0 \\
\text { as } x_{i} \in P_{0}, y_{i} \in P_{1}(i=0,1),\left\|x_{0}\right\|+\left\|x_{1}\right\|+\left\|y_{0}\right\|+\left\|y_{1}\right\| \longrightarrow \infty,
\end{gathered}
$$

uniformly for $t \in J_{+}$, and

$\int_{0}^{\infty} a_{i}(t) \mathrm{d} t=a_{i}^{*}<\infty, \quad \int_{0}^{\infty} b_{i}(t) \mathrm{d} t=b_{i}^{*}<\infty, \quad \int_{0}^{\infty} c_{i}(t)(1+t) \mathrm{d} t=c_{i}^{*}<\infty \quad(i=0,1)$.

$\left(\mathrm{H}_{2}\right)$ For any $t \in J_{+}, R>0$ and countable bounded set $V_{i} \subset D C^{1}\left[J, P_{0 R}^{*}\right], W_{i} \subset$ $D C^{1}\left[J, P_{1 R}^{*}\right](i=0,1)$, there exist $L_{i j}(t), K_{i j}(t) \in L[J, J](i, j=0,1)$ such that

$$
\begin{aligned}
& \alpha\left(f\left(t, V_{0}(t), V_{1}(t), W_{0}(t), W_{1}(t)\right)\right) \leq \sum_{i=0}^{1} L_{0 i}(t) \alpha\left(V_{i}(t)\right)+K_{0 i}(t) \alpha\left(W_{i}(t)\right), \\
& \alpha\left(g\left(t, V_{0}(t), V_{1}(t), W_{0}(t), W_{1}(t)\right)\right) \leq \sum_{i=0}^{1} L_{1 i}(t) \alpha\left(V_{i}(t)\right)+K_{1 i}(t) \alpha\left(W_{i}(t)\right),
\end{aligned}
$$

with

$$
\left(D_{i}+1\right) \int_{0}^{+\infty}\left[\left(L_{i 0}(s)+K_{i 0}(s)\right)(1+s)+L_{i 1}(s)+K_{i 1}(s)\right] \mathrm{d} s<\frac{1}{2} \quad(i=0,1)
$$

where $P_{0 R}^{*}=\left\{x \in P, x \geq x_{0}^{*},\|x\| \leq R\right\}, P_{1 R}^{*}=\left\{y \in P, y \geq y_{0}^{*},\|y\| \leq R\right\}$.

$\left(\mathrm{H}_{3}\right) t \in J_{+}, x_{0}^{*} \leq x_{i} \leq \bar{x}_{i}, y_{0}^{*} \leq y_{i} \leq \bar{y}_{i}(i=0,1)$ imply

$$
\begin{aligned}
& f\left(t, x_{0}, x_{1}, y_{0}, y_{1}\right) \leq f\left(t, \bar{x}_{0}, \bar{x}_{1}, \bar{y}_{0}, \bar{y}_{1}\right), \\
& g\left(t, x_{0}, x_{1}, y_{0}, y_{1}\right) \leq g\left(t, \bar{x}_{0}, \bar{x}_{1}, \bar{y}_{0}, \bar{y}_{1}\right) .
\end{aligned}
$$

In what follows, we write $Q_{1}=\left\{x \in D C^{1}[J, P]: x^{(i)}(t) \geq x_{0}^{*}, \forall t \in J, i=0,1\right\}, Q_{2}=\{y \in$ $\left.D C^{1}[J, P]: y^{(i)}(t) \geq y_{0}^{*}, \forall t \in J, i=0,1\right\}$, and $Q=Q_{1} \times Q_{2}$. Evidently, $Q_{1}, Q_{2}$, and $Q$ are closed convex sets in $D C^{1}[J, E]$ and $X$, respectively. 
We will reduce BVP (1.2) to a system of integral equations in $E$. To this end, we first consider operator $A$ defined by

$$
A(x, y)(t)=\left(A_{1}(x, y)(t), A_{2}(x, y)(t)\right)
$$

where

$$
\begin{aligned}
A_{1}(x, y)(t)= & \frac{1}{1-\sum_{i=1}^{m-2} \alpha_{i}}\left[\left(\sum_{i=1}^{m-2} \alpha_{i} \xi_{i}\right) x_{\infty}+\sum_{i=1}^{m-2} \alpha_{i} \int_{0}^{\xi_{i}} \int_{s}^{+\infty} f\left(\tau, x(\tau), x^{\prime}(\tau), y(\tau), y^{\prime}(\tau)\right) \mathrm{d} \tau \mathrm{d} s\right] \\
& +\int_{0}^{t} \int_{s}^{+\infty} f\left(\tau, x(\tau), x^{\prime}(\tau), y(\tau), y^{\prime}(\tau)\right) \mathrm{d} \tau \mathrm{d} s+t x_{\infty}, \\
A_{2}(x, y)(t)= & \frac{1}{1-\sum_{i=1}^{m-2} \beta_{i}}\left[\left(\sum_{i=1}^{m-2} \beta_{i} \xi_{i}\right) y_{\infty}+\sum_{i=1}^{m-2} \beta_{i} \int_{0}^{\xi_{i}} \int_{s}^{+\infty} g\left(\tau, x(\tau), x^{\prime}(\tau), y(\tau), y^{\prime}(\tau)\right) \mathrm{d} \tau \mathrm{d} s\right] \\
& +\int_{0}^{t} \int_{s}^{+\infty} g\left(\tau, x(\tau), x^{\prime}(\tau), y(\tau), y^{\prime}(\tau)\right) \mathrm{d} \tau \mathrm{d} s+t y_{\infty} .
\end{aligned}
$$

Lemma 2.1. If condition $\left(H_{1}\right)$ is satisfied, then operator A defined by (2.12) is a continuous operator from $Q$ into $Q$.

Proof. Let

$$
\begin{gathered}
\epsilon_{0}=\min \left\{\frac{1}{8 c_{0}^{*}\left(1+\sum_{i=1}^{m-2} \alpha_{i} \xi_{m-2} /\left(1-\sum_{i=1}^{m-2} \alpha_{i}\right)\right)}, \frac{1}{8 c_{1}^{*}\left(1+\sum_{i=1}^{m-2} \beta_{i} \xi_{m-2} /\left(1-\sum_{i=1}^{m-2} \beta_{i}\right)\right)}\right\}, \\
r=\min \left\{\frac{\left\|x_{0}^{*}\right\|}{N}, \frac{\left\|y_{0}^{*}\right\|}{N}\right\}>0 .
\end{gathered}
$$

By virtue of condition $\left(\mathrm{H}_{1}\right)$, there exists an $R>r$ such that

$\left\|f\left(t, x_{0}, x_{1}, y_{0}, y_{1}\right)\right\| \leq \epsilon_{0} c_{0}(t)\left(\left\|x_{0}\right\|+\left\|x_{1}\right\|+\left\|y_{0}\right\|+\left\|y_{1}\right\|\right), \quad \forall t \in J_{+}, x_{i} \in P_{0}, y_{i} \in P_{1}(i=0,1)$,

$$
\left\|x_{0}\right\|+\left\|x_{1}\right\|+\left\|y_{0}\right\|+\left\|y_{1}\right\|>R,
$$

$\left\|f\left(t, x_{0}, x_{1}, y_{0}, y_{1}\right)\right\| \leq a_{0}(t)+M_{0} b_{0}(t), \quad \forall t \in J_{+}, x_{i} \in P_{0}, y_{i} \in P_{1}(i=0,1)$,

$$
\left\|x_{0}\right\|+\left\|x_{1}\right\|+\left\|y_{0}\right\|+\left\|y_{1}\right\| \leq R,
$$


where

$$
M_{0}=\max \left\{h_{0}\left(u_{0}, u_{1}, v_{0}, v_{1}\right): r \leq u_{i}, v_{i} \leq R(i=0,1)\right\} .
$$

Hence

$$
\begin{array}{r}
\left\|f\left(t, x_{0}, x_{1}, y_{0}, y_{1}\right)\right\| \leq \epsilon_{0} c_{0}(t)\left(\left\|x_{0}\right\|+\left\|x_{1}\right\|+\left\|y_{0}\right\|+\left\|y_{1}\right\|\right)+a_{0}(t)+M_{0} b_{0}(t) \\
\forall t \in J_{+}, x_{i} \in P_{0}, y_{i} \in P_{1}(i=0,1) .
\end{array}
$$

Let $(x, y) \in Q$, we have, by $(2.19)$

$$
\begin{aligned}
\| f(t, & \left.x(t), x^{\prime}(t), y(t), y^{\prime}(t)\right) \| \\
& \leq \epsilon_{0} c_{0}(t)(1+t)\left(\frac{\|x(t)\|}{t+1}+\frac{\left\|x^{\prime}(t)\right\|}{t+1}+\frac{\|y(t)\|}{t+1}+\frac{\left\|y^{\prime}(t)\right\|}{t+1}\right)+a_{0}(t)+M_{0} b_{0}(t) \\
& \leq \epsilon_{0} c_{0}(t)(1+t)\left(\|x\|_{F}+\left\|x^{\prime}\right\|_{C}+\|y\|_{F}+\left\|y^{\prime}\right\|_{C}\right)+a_{0}(t)+M_{0} b_{0}(t) \\
& \leq 2 \epsilon_{0} c_{0}(t)(1+t)\left(\|x\|_{D}+\|y\|_{D}\right)+a_{0}(t)+M_{0} b_{0}(t) \\
& \leq 4 \epsilon_{0} c_{0}(t)(1+t)\|(x, y)\|_{X}+a_{0}(t)+M_{0} b_{0}(t), \quad \forall t \in J_{+},
\end{aligned}
$$

which together with condition $\left(\mathrm{H}_{2}\right)$ implies the convergence of the infinite integral

$$
\int_{0}^{\infty}\left\|f\left(s, x(s), x^{\prime}(s), y(s), y^{\prime}(s)\right)\right\| \mathrm{d} s
$$

Thus, we have

$$
\begin{aligned}
\left\|\int_{0}^{t} \int_{s}^{+\infty} f\left(\tau, x(\tau), x^{\prime}(\tau), y(\tau), y^{\prime}(\tau)\right) \mathrm{d} \tau \mathrm{d} s\right\| & \leq \int_{0}^{t} \int_{s}^{+\infty}\left\|f\left(\tau, x(\tau), x^{\prime}(\tau), y(\tau), y^{\prime}(\tau)\right)\right\| \mathrm{d} \tau \mathrm{d} s \\
& \leq \int_{0}^{+\infty} \int_{0}^{t}\left\|f\left(\tau, x(\tau), x^{\prime}(\tau), y(\tau), y^{\prime}(\tau)\right)\right\| \mathrm{d} s \mathrm{~d} \tau \\
& \leq t \int_{0}^{\infty}\left\|f\left(s, x(s), x^{\prime}(s), y(s), y^{\prime}(s)\right)\right\| \mathrm{d} s, \quad \forall t \in J_{+},
\end{aligned}
$$


which together with (2.13) and $\left(\mathrm{H}_{1}\right)$ implies that

$$
\begin{aligned}
\left\|A_{1}(x, y)(t)\right\| \leq & \int_{0}^{t} \int_{s}^{+\infty}\left\|f\left(\tau, x(\tau), x^{\prime}(\tau), y(\tau), y^{\prime}(\tau)\right)\right\| \mathrm{d} \tau \mathrm{d} s+t\left\|x_{\infty}\right\|+\frac{\sum_{i=1}^{m-2} \alpha_{i} \xi_{i}}{1-\sum_{i=1}^{m-2} \alpha_{i}}\left\|x_{\infty}\right\| \\
& +\frac{1}{1-\sum_{i=1}^{m-2} \alpha_{i}} \sum_{i=1}^{m-2} \alpha_{i} \int_{0}^{\xi_{m-2}} \int_{s}^{+\infty}\left\|f\left(\tau, x(\tau), x^{\prime}(\tau), y(\tau), y^{\prime}(\tau)\right)\right\| \mathrm{d} \tau \mathrm{d} s \\
\leq & t\left(\int_{0}^{+\infty}\left\|f\left(\tau, x(\tau), x^{\prime}(\tau), y(\tau), y^{\prime}(\tau)\right)\right\| \mathrm{d} \tau+\left\|x_{\infty}\right\|\right)+\frac{\sum_{i=1}^{m-2} \alpha_{i} \xi_{i}}{1-\sum_{i=1}^{m-2} \alpha_{i}}\left\|x_{\infty}\right\| \\
& +\frac{1}{1-\sum_{i=1}^{m-2} \alpha_{i}} \sum_{i=1}^{m-2} \alpha_{i} \xi_{m-2}\left(\int_{0}^{+\infty}\left\|f\left(\tau, x(\tau), x^{\prime}(\tau), y(\tau), y^{\prime}(\tau)\right)\right\| \mathrm{d} \tau\right) .
\end{aligned}
$$

Therefore, by (2.15) and (2.20), we get

$$
\begin{aligned}
\frac{\left\|A_{1}(x, y)(t)\right\|}{1+t} \leq & \int_{0}^{+\infty}\left\|f\left(\tau, x(\tau), x^{\prime}(\tau), y(\tau), y^{\prime}(\tau)\right)\right\| \mathrm{d} \tau+\left\|x_{\infty}\right\|+\frac{\sum_{i=1}^{m-2} \alpha_{i} \xi_{i}}{1-\sum_{i=1}^{m-2} \alpha_{i}}\left\|x_{\infty}\right\| \\
& +\frac{1}{1-\sum_{i=1}^{m-2} \alpha_{i}} \sum_{i=1}^{m-2} \alpha_{i} \xi_{m-2}\left(\int_{0}^{+\infty}\left\|f\left(\tau, x(\tau), x^{\prime}(\tau), y(\tau), y^{\prime}(\tau)\right)\right\| \mathrm{d} \tau\right) \\
\leq & \left(1+\frac{1}{1-\sum_{i=1}^{m-2} \alpha_{i}} \sum_{i=1}^{m-2} \alpha_{i} \xi_{m-2}\right)\left[4 \epsilon_{0} c_{0}^{*}\|(x, y)\|_{X}+a_{0}^{*}+M b_{0}^{*}\right] \\
& +\left(1+\frac{\sum_{i=1}^{m-2} \alpha_{i} \xi_{i}}{1-\sum_{i=1}^{m-2} \alpha_{i}}\right)\left\|x_{\infty}\right\| \\
\leq & \frac{1}{2}\|(x, y)\|_{X}+\left(1+\frac{1}{1-\sum_{i=1}^{m-2} \alpha_{i}} \sum_{i=1}^{m-2} \alpha_{i} \xi_{m-2}\right)\left(a_{0}^{*}+M b_{0}^{*}\right) \\
& +\left(1+\frac{\sum_{i=1}^{m-2} \alpha_{i} \xi_{i}}{1-\sum_{i=1}^{m-2} \alpha_{i}}\right)\left\|x_{\infty}\right\| .
\end{aligned}
$$

Differentiating (2.13), we obtain

$$
A_{1}^{\prime}(x, y)(t)=\int_{t}^{+\infty} f\left(s, x(s), x^{\prime}(s), y(s), y^{\prime}(s)\right) \mathrm{d} s+x_{\infty}
$$


Hence,

$$
\begin{aligned}
\left\|A_{1}^{\prime}(x, y)(t)\right\| & \leq \int_{0}^{+\infty}\left\|f\left(s, x(s), x^{\prime}(s), y(s), y^{\prime}(s)\right)\right\| \mathrm{d} s+\left\|x_{\infty}\right\| \\
& \leq 4 \epsilon_{0} c_{0}^{*}\|(x, y)\|_{X}+a_{0}^{*}+M_{0} b_{0}^{*}+\left\|x_{\infty}\right\| \\
& \leq \frac{1}{2}\|(x, y)\|_{X}+a_{0}^{*}+M_{0} b_{0}^{*}+\left\|x_{\infty}\right\|, \quad \forall t \in J .
\end{aligned}
$$

It follows from (2.24) and (2.25) that

$$
\left\|A_{1}(x, y)\right\|_{D} \leq \frac{1}{2}\|(x, y)\|_{X}+\left(1+\frac{\sum_{i=1}^{m-2} \alpha_{i} \xi_{m-2}}{1-\sum_{i=1}^{m-2} \alpha_{i}}\right)\left(a_{0}^{*}+M_{0} b_{0}^{*}\right)+\left(1+\frac{\sum_{i=1}^{m-2} \alpha_{i} \xi_{i}}{1-\sum_{i=1}^{m-2} \alpha_{i}}\right)\left\|x_{\infty}\right\| .
$$

So, $A_{1}(x, y) \in D C^{1}[J, E]$. On the other hand, it can be easily seen that

$$
\begin{gathered}
A_{1}(x, y)(t) \geq \frac{\sum_{i=1}^{m-2} \alpha_{i} \xi_{i}}{1-\sum_{i=1}^{m-2} \alpha_{i}} x_{\infty} \geq x_{\infty} \geq x_{0}^{*}, \quad \forall t \in J, \\
A_{1}^{\prime}(x, y)(t) \geq x_{\infty} \geq x_{0}^{*}, \quad \forall t \in J .
\end{gathered}
$$

So, $A_{1}(x, y) \in Q_{1}$. In the same way, we can easily get that

$$
\begin{gathered}
\left\|A_{2}(x, y)\right\|_{D} \leq \frac{1}{2}\|(x, y)\|_{X}+\left(1+\frac{\sum_{i=1}^{m-2} \beta_{i} \xi_{m-2}}{1-\sum_{i=1}^{m-2} \beta_{i}}\right)\left(a_{1}^{*}+M_{1} b_{1}^{*}\right)+\left(1+\frac{\sum_{i=1}^{m-2} \beta_{i} \xi_{i}}{1-\sum_{i=1}^{m-2} \beta_{i}}\right)\left\|y_{\infty}\right\|, \\
A_{2}(x, y)(t) \geq \frac{\sum_{i=1}^{m-2} \beta_{i} \xi_{i}}{1-\sum_{i=1}^{m-2} \beta_{i}} y_{\infty} \geq y_{\infty} \geq y_{0}^{*}, \quad \forall t \in J, \\
A_{2}^{\prime}(x, y)(t) \geq y_{\infty} \geq y_{0}^{*}, \quad \forall t \in J,
\end{gathered}
$$

where $M_{1}=\max \left\{h_{1}\left(u_{0}, u_{1}, v_{0}, v_{1}\right): r \leq u_{i}, v_{i} \leq R(i=0,1)\right\}$. Thus, $A$ maps $Q$ into $Q$ and we get

$$
\|A(x, y)\|_{X} \leq \frac{1}{2}\|(x, y)\|_{X}+\gamma
$$

where

$$
\begin{aligned}
r=\max \{ & \left(1+\frac{\sum_{i=1}^{m-2} \alpha_{i} \xi_{m-2}}{1-\sum_{i=1}^{m-2} \alpha_{i}}\right)\left(a_{0}^{*}+M b_{0}^{*}\right)+\left(1+\frac{\sum_{i=1}^{m-2} \alpha_{i} \xi_{i}}{1-\sum_{i=1}^{m-2} \alpha_{i}}\right)\left\|x_{\infty}\right\|, \\
& \left.\left(1+\frac{\sum_{i=1}^{m-2} \beta_{i} \xi_{m-2}}{1-\sum_{i=1}^{m-2} \beta_{i}}\right)\left(a_{1}^{*}+M_{1} b_{1}^{*}\right)+\left(1+\frac{\sum_{i=1}^{m-2} \beta_{i} \xi_{i}}{1-\sum_{i=1}^{m-2} \beta_{i}}\right)\left\|y_{\infty}\right\|\right\} .
\end{aligned}
$$


Finally, we show that $A$ is continuous. Let $\left(x_{m}, y_{m}\right),(\bar{x}, \bar{y}) \in Q,\left\|\left(x_{m}, y_{m}\right)-(\bar{x}, \bar{y})\right\|_{X} \rightarrow$ $0(m \rightarrow \infty)$. Then $\left\{\left(x_{m}, y_{m}\right)\right\}$ is a bounded subset of $Q$. Thus, there exists $r>0$ such that $\sup _{m}\left\|\left(x_{m}, y_{m}\right)\right\|_{X}<r$ for $m \geq 1$ and $\|(\bar{x}, \bar{y})\|_{X} \leq r+1$. Similar to (2.24) and (2.26), it is easy to have

$$
\begin{aligned}
& \left\|A_{1}\left(x_{m}, y_{m}\right)-A_{1}(\bar{x}, \bar{y})\right\|_{X} \\
& \quad \leq \int_{0}^{+\infty}\left\|f\left(s, x_{m}(s), x_{m}^{\prime}(s), y_{m}(s), y_{m}^{\prime}(s)\right)-f\left(s, \bar{x}(s), \overline{x^{\prime}}(s), \bar{y}(s), \overline{y^{\prime}}(s)\right)\right\| \mathrm{d} s \\
& \quad+\frac{\sum_{i=1}^{m-2} \alpha_{i} \xi_{m-2}}{1-\sum_{i=1}^{m-2} \alpha_{i}} \int_{0}^{+\infty}\left\|f\left(s, x_{m}(s), x_{m}^{\prime}(s), y_{m}(s), y_{m}^{\prime}(s)\right)-f\left(s, \bar{x}(s), \bar{x}^{\prime}(s), \bar{y}(s), \bar{y}^{\prime}(s)\right)\right\| \mathrm{d} s .
\end{aligned}
$$

It is clear,

$$
f\left(t, x_{m}(t), x_{m}^{\prime}(t), y_{m}(t), y_{m}^{\prime}(t)\right) \longrightarrow f\left(t, \bar{x}(t), \bar{x}^{\prime}(t), \bar{y}(t), \bar{y}^{\prime}(t)\right) \quad \text { as } m \longrightarrow \infty, \forall t \in J_{+},
$$

and by (2.20),

$$
\begin{aligned}
& \left\|f\left(t, x_{m}(t), x_{m}^{\prime}(t), y_{m}(t), y_{m}^{\prime}(t)\right)-f\left(t, \bar{x}(t), \bar{x}^{\prime}(t), \bar{y}(t), \bar{y}^{\prime}(t)\right)\right\| \\
& \quad \leq 8 \epsilon_{0} c_{0}(t)(1+t) r+2 a_{0}(t)+2 M_{0} b_{0}(t) \\
& \quad=\sigma_{0}(t) \in L[J, J], \quad m=1,2,3, \ldots, \forall t \in J_{+} .
\end{aligned}
$$

It follows from (2.33) and (2.34) and the dominated convergence theorem that

$$
\lim _{m \rightarrow \infty} \int_{0}^{\infty}\left\|f\left(s, x_{m}(s), x_{m}^{\prime}(s), y_{m}(s), y_{m}^{\prime}(s)\right)-f\left(s, \bar{x}(s), \bar{x}^{\prime}(s), \bar{y}(s), \bar{y}^{\prime}(s)\right)\right\| \mathrm{d} s=0
$$

It follows from (2.32) and (2.35) that $\left\|A_{1}\left(x_{m}, y_{m}\right)-A_{1}(\bar{x}, \bar{y})\right\|_{D} \rightarrow 0$ as $m \rightarrow \infty$. By the same method, we have $\left\|A_{2}\left(x_{m}, y_{m}\right)-A_{2}(\bar{x}, \bar{y})\right\|_{D} \rightarrow 0$ as $m \rightarrow \infty$. Therefore, the continuity of $A$ is proved.

Lemma 2.2. If condition $\left(H_{1}\right)$ is satisfied, then $(x, y) \in Q \cap\left(C^{2}\left[J_{+}, E\right] \times C^{2}\left[J_{+}, E\right]\right)$ is a solution of $B V P(1.2)$ if and only if $(x, y) \in Q$ is a fixed point of operator $A$.

Proof. Suppose that $x \in Q \cap\left(C^{2}\left[J_{+}, E\right] \times C^{2}\left[J_{+}, E\right]\right)$ is a solution of BVP (1.2). For $t \in J$, integrating (1.2) from $t$ to $+\infty$, we have

$$
\begin{aligned}
& x^{\prime}(t)=x_{\infty}+\int_{t}^{+\infty} f\left(s, x(s), x^{\prime}(s), y(s), y^{\prime}(s)\right) \mathrm{d} s, \\
& y^{\prime}(t)=y_{\infty}+\int_{t}^{+\infty} g\left(s, x(s), x^{\prime}(s), y(s), y^{\prime}(s)\right) \mathrm{d} s .
\end{aligned}
$$


Integrating (2.36) from 0 to $t$, we get

$$
\begin{aligned}
& x(t)=x(0)+t x_{\infty}+\int_{0}^{t} \int_{s}^{+\infty} f\left(\tau, x(\tau), x^{\prime}(\tau), y(\tau), y^{\prime}(\tau)\right) \mathrm{d} \tau \mathrm{d} s, \\
& y(t)=y(0)+t y_{\infty}+\int_{0}^{t} \int_{s}^{+\infty} g\left(\tau, x(\tau), x^{\prime}(\tau), y(\tau), y^{\prime}(\tau)\right) \mathrm{d} \tau \mathrm{d} s .
\end{aligned}
$$

Thus, we obtain

$$
\begin{aligned}
& x\left(\xi_{i}\right)=x(0)+\xi_{i} x_{\infty}+\int_{0}^{\xi_{i}} \int_{s}^{+\infty} f\left(\tau, x(\tau), x^{\prime}(\tau), y(\tau), y^{\prime}(\tau)\right) \mathrm{d} \tau \mathrm{d} s \\
& y\left(\xi_{i}\right)=y(0)+\xi_{i} y_{\infty}+\int_{0}^{\xi_{i}} \int_{s}^{+\infty} g\left(\tau, x(\tau), x^{\prime}(\tau), y(\tau), y^{\prime}(\tau)\right) \mathrm{d} \tau \mathrm{d} s
\end{aligned}
$$

which together with the boundary value conditions imply that

$$
\begin{aligned}
& x(0)=\frac{1}{1-\sum_{i=1}^{m-2} \alpha_{i}}\left[\left(\sum_{i=1}^{m-2} \alpha_{i} \xi_{i}\right) x_{\infty}+\sum_{i=1}^{m-2} \alpha_{i} \int_{0}^{\xi} \int_{s}^{+\infty} f\left(\tau, x(\tau), x^{\prime}(\tau), y(\tau), y^{\prime}(\tau)\right) \mathrm{d} \tau \mathrm{d} s\right], \\
& y(0)=\frac{1}{1-\sum_{i=1}^{m-2} \beta_{i}}\left[\left(\sum_{i=1}^{m-2} \beta_{i} \xi_{i}\right) y_{\infty}+\sum_{i=1}^{m-2} \beta_{i} \int_{0}^{\xi} \int_{s}^{+\infty} g\left(\tau, x(\tau), x^{\prime}(\tau), y(\tau), y^{\prime}(\tau)\right) \mathrm{d} \tau \mathrm{d} s\right] .
\end{aligned}
$$

Substituting (2.40) and (2.41) into (2.37) and (2.38), respectively, we have

$$
\begin{aligned}
x(t)= & \frac{1}{1-\sum_{i=1}^{m-2} \alpha_{i}}\left[\left(\sum_{i=1}^{m-2} \alpha_{i} \xi_{i}\right) x_{\infty}+\sum_{i=1}^{m-2} \alpha_{i} \int_{0}^{\xi_{i}} \int_{s}^{+\infty} f\left(\tau, x(\tau), x^{\prime}(\tau), y(\tau), y^{\prime}(\tau)\right) \mathrm{d} \tau \mathrm{d} s\right] \\
& +\int_{0}^{t} \int_{s}^{+\infty} f\left(\tau, x(\tau), x^{\prime}(\tau), y(\tau), y^{\prime}(\tau)\right) \mathrm{d} \tau \mathrm{d} s+t x_{\infty}, \\
y(t)= & \frac{1}{1-\sum_{i=1}^{m-2} \beta_{i}}\left[\left(\sum_{i=1}^{m-2} \beta_{i} \xi_{i}\right) y_{\infty}+\sum_{i=1}^{m-2} \beta_{i} \int_{0}^{\xi_{i}} \int_{s}^{+\infty} g\left(\tau, x(\tau), x^{\prime}(\tau), y(\tau), y^{\prime}(\tau)\right) \mathrm{d} \tau \mathrm{d} s\right] \\
& +\int_{0}^{t} \int_{s}^{+\infty} g\left(\tau, x(\tau), x^{\prime}(\tau), y(\tau), y^{\prime}(\tau)\right) \mathrm{d} \tau \mathrm{d} s+t y_{\infty} .
\end{aligned}
$$

It follows from Lemma 2.1 that the integral $\int_{0}^{t} \int_{s}^{+\infty} f\left(\tau, x(\tau), x^{\prime}(\tau), y(\tau), y^{\prime}(\tau)\right) \mathrm{d} \tau \mathrm{d} s$ and the integral $\int_{0}^{t} \int_{s}^{+\infty} g\left(\tau, x(\tau), x^{\prime}(\tau), y(\tau), y^{\prime}(\tau)\right) \mathrm{d} \tau \mathrm{d} s$ are convergent. Thus, $(x, y)$ is a fixed point of operator $A$. 

proof.

Conversely, if $(x, y)$ is fixed point of operator $A$, then direct differentiation gives the

Lemma 2.3. Let $\left(H_{1}\right)$ be satisfied, $V \subset Q$ is a bounded set. Then $\left(A_{i} V\right)(t) /(1+t)$ and $\left(A_{i}^{\prime} V\right)(t)$ are equicontinuous on any finite subinterval of $J$ and for any $\epsilon>0$, there exists $N_{i}>0$ such that

$$
\left\|\frac{A_{i}(x, y)\left(t_{1}\right)}{1+t_{1}}-\frac{A_{i}(x, y)\left(t_{2}\right)}{1+t_{2}}\right\|<\epsilon, \quad\left\|A_{i}^{\prime}(x, y)\left(t_{1}\right)-A_{i}^{\prime}(x, y)\left(t_{2}\right)\right\|<\epsilon
$$

uniformly with respect to $(x, y) \in V$ as $t_{1}, t_{2} \geq N_{i}(i=1,2)$.

Proof. We only give the proof for operator $A_{1}$, the proof for operator $A_{2}$ can be given in a similar way. By (2.13), we have

$$
\begin{aligned}
A_{1}(x, y)(t)= & \frac{1}{1-\sum_{i=1}^{m-2} \alpha_{i}}\left[\left(\sum_{i=1}^{m-2} \alpha_{i} \xi_{i}\right) x_{\infty}+\sum_{i=1}^{m-2} \alpha_{i} \int_{0}^{\xi_{i}} \int_{s}^{+\infty} f\left(\tau, x(\tau), x^{\prime}(\tau), y(\tau), y^{\prime}(\tau)\right) \mathrm{d} \tau \mathrm{d} s\right] \\
& +\int_{0}^{t} \int_{s}^{+\infty} f\left(\tau, x(\tau), x^{\prime}(\tau), y(\tau), y^{\prime}(\tau)\right) \mathrm{d} \tau \mathrm{d} s+t x_{\infty} \\
= & \frac{1}{1-\sum_{i=1}^{m-2} \alpha_{i}}\left[\left(\sum_{i=1}^{m-2} \alpha_{i} \xi_{i}\right) x_{\infty}+\sum_{i=1}^{m-2} \alpha_{i} \int_{0}^{\xi_{i}} \int_{s}^{+\infty} f\left(\tau, x(\tau), x^{\prime}(\tau), y(\tau), y^{\prime}(\tau)\right) \mathrm{d} \tau \mathrm{d} s\right] \\
& +t x_{\infty}+t \int_{t}^{+\infty} f\left(s, x(s), x^{\prime}(s), y(s), y^{\prime}(s)\right) \mathrm{d} s+\int_{0}^{t} s f\left(s, x(s), x^{\prime}(s), y(s), y^{\prime}(s)\right) \mathrm{d} s .
\end{aligned}
$$

For $(x, y) \in V, t_{2}>t_{1}$, we obtain by $(2.44)$

$$
\begin{aligned}
& \left\|\frac{A_{1}(x, y)\left(t_{1}\right)}{1+t_{1}}-\frac{A_{1}(x, y)\left(t_{2}\right)}{1+t_{2}}\right\| \\
& \leq\left|\frac{1}{1+t_{1}}-\frac{1}{1+t_{2}}\right| \cdot \frac{1}{1-\sum_{i=1}^{m-2} \alpha_{i}} \\
& \quad \times\left[\left(\sum_{i=1}^{m-2} \alpha_{i} \xi_{i}\right)\left\|x_{\infty}\right\|+\sum_{i=1}^{m-2} \alpha_{i} \int_{0}^{\xi_{i}} \int_{s}^{+\infty} f\left(\tau, x(\tau), x^{\prime}(\tau), y(\tau), y^{\prime}(\tau)\right) \mathrm{d} \tau \mathrm{d} s\right] \\
& +\left|\frac{t_{1}}{1+t_{1}}-\frac{t_{2}}{1+t_{2}}\right| \cdot\left\|x_{\infty}\right\| \\
& +\left\|\frac{t_{1}}{1+t_{1}} \int_{t_{1}}^{+\infty} f\left(s, x(s), x^{\prime}(s), y(s), y^{\prime}(s)\right) \mathrm{d} s-\frac{t_{2}}{1+t_{2}} \int_{t_{2}}^{+\infty} f\left(s, x(s), x^{\prime}(s), y(s), y^{\prime}(s)\right) \mathrm{d} s\right\|
\end{aligned}
$$




$$
\begin{aligned}
& +\left\|\int_{0}^{t_{1}} \frac{s}{1+t_{1}} f\left(s, x(s), x^{\prime}(s), y(s), y^{\prime}(s)\right) \mathrm{d} s-\int_{0}^{t_{2}} \frac{s}{1+t_{2}} f\left(s, x(s), x^{\prime}(s), y(s), y^{\prime}(s)\right) \mathrm{d} s\right\| \\
& \leq\left|\frac{1}{1+t_{1}}-\frac{1}{1+t_{2}}\right| \cdot \frac{1}{1-\sum_{i=1}^{m-2} \alpha_{i}}{ }^{\times}\left[\left(\sum_{i=1}^{m-2} \alpha_{i} \xi_{i}\right)\left\|x_{\infty}\right\|+\sum_{i=1}^{m-2} \alpha_{i} \int_{0}^{\xi_{i}} \int_{s}^{+\infty} f\left(\tau, x(\tau), x^{\prime}(\tau), y(\tau), y^{\prime}(\tau)\right) \mathrm{d} \tau \mathrm{d} s\right] \\
& +\left|\frac{t_{1}}{1+t_{1}}-\frac{t_{2}}{1+t_{2}}\right| \cdot\left\|x_{\infty}\right\|+\left|\frac{t_{1}}{1+t_{1}}-\frac{t_{2}}{1+t_{2}}\right| \cdot\left\|\int_{0}^{+\infty} f\left(s, x(s), x^{\prime}(s), y(s), y^{\prime}(s)\right) \mathrm{d} s\right\| \\
& +\left|\frac{t_{1}}{1+t_{1}}-\frac{t_{2}}{1+t_{2}}\right| \cdot\left\|\int_{0}^{t_{1}} f\left(s, x(s), x^{\prime}(s), y(s), y^{\prime}(s)\right) \mathrm{d} s\right\| \\
& +\frac{t_{2}}{1+t_{2}}\left\|\int_{t_{1}}^{t_{2}} f\left(s, x(s), x^{\prime}(s), y(s), y^{\prime}(s)\right) \mathrm{d} s\right\| \\
& +\left|\frac{1}{1+t_{1}}-\frac{1}{1+t_{2}}\right| \cdot\left\|\int_{0}^{t_{1}} s f\left(s, x(s), x^{\prime}(s), y(s), y^{\prime}(s)\right) \mathrm{d} s\right\| \\
& +\left\|\int_{t_{1}}^{t_{2}} s f\left(s, x(s), x^{\prime}(s), y(s), y^{\prime}(s)\right) \mathrm{d} s\right\| .
\end{aligned}
$$

Then, it is easy to see by $(2.45)$ and $\left(\mathrm{H}_{1}\right)$ that $\left\{A_{1} V(t) /(1+t)\right\}$ is equicontinuous on any finite subinterval of $J$.

Since $V \subset Q$ is bounded, there exists $r>0$ such that for any $(x, y) \in V,\|(x, y)\|_{X} \leq r$. By (2.25), we get

$$
\begin{aligned}
\left\|A_{1}^{\prime}(x, y)\left(t_{1}\right)-A_{1}^{\prime}(x, y)\left(t_{2}\right)\right\| & =\left\|\int_{t_{1}}^{t_{2}} f\left(s, x(s), x^{\prime}(s), y(s), y^{\prime}(s)\right) \mathrm{d} s\right\| \\
& \leq \int_{t_{1}}^{t_{2}}\left[4 \epsilon_{0} r c(s)(1+s)+a_{0}(s)+M_{0} b_{0}(s)\right] \mathrm{d} s .
\end{aligned}
$$

It follows from (2.46) and $\left(\mathrm{H}_{1}\right)$ and the absolute continuity of Lebesgue integral that $\left\{A_{1}^{\prime} V(t)\right\}$ is equicontinuous on any finite subinterval of $J$. that

In the following, we are in position to show that for any $\epsilon>0$, there exists $N_{1}>0$ such

$$
\left\|\frac{A_{1}(x, y)\left(t_{1}\right)}{1+t_{1}}-\frac{A_{1}(x, y)\left(t_{2}\right)}{1+t_{2}}\right\|<\epsilon, \quad\left\|A_{1}^{\prime}(x, y)\left(t_{1}\right)-A_{1}^{\prime}(x, y)\left(t_{2}\right)\right\|<\epsilon
$$

uniformly with respect to $x \in V$ as $t_{1}, t_{2} \geq N$. 
Combining with (2.45), we need only to show that for any $\epsilon>0$, there exists sufficiently large $N>0$ such that

$$
\left\|\int_{0}^{t_{1}} \frac{s}{1+t_{1}} f\left(s, x(s), x^{\prime}(s), y(s), y^{\prime}(s)\right) \mathrm{d} s-\int_{0}^{t_{2}} \frac{s}{1+t_{2}} f\left(s, x(s), x^{\prime}(s), y(s), y^{\prime}(s)\right) \mathrm{d} s\right\|<\epsilon
$$

for all $x \in V$ as $t_{1}, t_{2} \geq N$. The rest part of the proof is very similar to Lemma 2.3 in [5], we omit the details.

Lemma 2.4. Let $V$ be a bounded set in $D C^{1}[J, E] \times D C^{1}[J, E]$. Assume that $\left(H_{1}\right)$ holds. Then

$$
\alpha_{D}\left(A_{i} V\right)=\max \left\{\sup _{t \in J} \alpha\left(\frac{\left(A_{i} V\right)(t)}{1+t}\right), \sup _{t \in J} \alpha\left(\left(A_{i} V\right)^{\prime}(t)\right)\right\} .
$$

Proof. The proof is similar to that of Lemma 2.4 in [5], we omit it.

Lemma 2.5 (see $[1,2]$ ). Mönch Fixed-Point Theorem. Let $Q$ be a closed convex set of $E$ and $u \in Q$. Assume that the continuous operator $F: Q \rightarrow Q$ has the following property: $V \subset Q$ countable, $V \subset \overline{\mathrm{CO}}(\{u\} \cup F(V)) \Rightarrow V$ is relatively compact. Then $F$ has a fixed point in $Q$.

Lemma 2.6. If $\left(H_{3}\right)$ is satisfied, then for $x, y \in Q, x^{(i)} \leq y^{(i)}, t \in J(i=0,1)$ imply that $(A x)^{(i)} \leq$ $(A y)^{(i)}, t \in J(i=0,1)$.

Proof. It is easy to see that this lemma follows from (2.13), (2.25), and condition $\left(\mathrm{H}_{3}\right)$. The proof is obvious.

Lemma 2.7 (see [16]). Let $E$ and $F$ are bounded sets in $E$, then

$$
\tilde{\alpha}(D \times F)=\max \{\alpha(D), \alpha(F)\},
$$

where $\tilde{\alpha}$ and $\alpha$ denote the Kuratowski measure of noncompactness in $E \times E$ and $E$, respectively.

Lemma 2.8 (see [16]). Let $P$ be normal (fully regular) in $E, \widetilde{P}=P \times P$, then $\widetilde{P}$ is normal (fully regular) in $E \times E$.

\section{Main Results}

Theorem 3.1. If conditions $\left(H_{1}\right)$ and $\left(H_{2}\right)$ are satisfied, then BVP (1.2) has a positive solution $(\bar{x}, \bar{y}) \in\left(D C^{1}[J, E] \cap C^{2}\left[J_{+}^{\prime}, E\right]\right) \times\left(D C^{1}[J, E] \cap C^{2}\left[J_{+}^{\prime}, E\right]\right)$ satisfying $(\bar{x})^{(i)}(t) \geq x_{0}^{*},(\bar{y})^{(i)}(t) \geq y_{0}^{*}$ for $t \in J(i=0,1)$.

Proof. By Lemma 2.1, operator $A$ defined by (2.13) is a continuous operator from $Q$ into $Q$, and, by Lemma 2.2, we need only to show that $A$ has a fixed point $(\bar{x}, \bar{y})$ in $Q$. Choose $R>2 \gamma$ and let $Q^{*}=\left\{(x, y) \in Q:\|(x, y)\|_{X} \leq R\right\}$. Obviously, $Q^{*}$ is a bounded closed convex set in space $D C^{1}[J, E] \times D C^{1}[J, E]$. It is easy to see that $Q^{*}$ is not empty since $\left((1+t) x_{\infty},(1+t) y_{\infty}\right) \in$ $Q^{*}$. It follows from (2.27) and (3.6) that $(x, y) \in Q^{*}$ implies $A(x, y) \in Q^{*}$, that is, $A$ maps $Q^{*}$ 
into $Q^{*}$. Let $V=\left\{\left(x_{m}, y_{m}\right): m=1,2, \ldots\right\} \subset Q^{*}$ satisfying $V \subset \overline{\mathrm{co}}\left\{\left\{\left(u_{0}, v_{0}\right)\right\} \cup A V\right\}$ for some $\left(u_{0}, v_{0}\right) \in Q^{*}$. Then $\left\|\left(x_{m}, y_{m}\right)\right\|_{X} \leq R$. We have, by (2.13) and (2.25),

$$
\begin{aligned}
& A_{1}\left(x_{m}, y_{m}\right)(t) \\
& =\frac{1}{1-\sum_{i=1}^{m-2} \alpha_{i}}\left[\left(\sum_{i=1}^{m-2} \alpha_{i} \xi_{i}\right) x_{\infty}+\sum_{i=1}^{m-2} \alpha_{i} \int_{0}^{\xi_{i}} \int_{s}^{+\infty} f\left(\tau, x_{m}(\tau), x_{m}^{\prime}(\tau), y_{m}(\tau), y_{m}^{\prime}(\tau)\right) \mathrm{d} \tau \mathrm{d} s\right] \\
& \quad+\int_{0}^{t} \int_{s}^{+\infty} f\left(\tau, x_{m}(\tau), x_{m}^{\prime}(\tau), y_{m}(\tau), y_{m}^{\prime}(\tau)\right) \mathrm{d} \tau \mathrm{d} s+t x_{\infty}, \\
& A_{1}^{\prime}\left(x_{m}, y_{m}\right)(t)=\int_{t}^{+\infty} f\left(s, x_{m}(s), x_{m}^{\prime}(s), y_{m}(s), y_{m}^{\prime}(s)\right) \mathrm{d} s+x_{\infty} .
\end{aligned}
$$

By Lemma 2.4, we have

$$
\alpha_{D}\left(A_{1} V\right)=\max \left\{\sup _{t \in J} \alpha\left(\left(A_{1} V\right)^{\prime}(t)\right), \sup _{t \in J} \alpha\left(\frac{\left(A_{1} V\right)(t)}{1+t}\right)\right\}
$$

where $A_{1} V(t)=\left\{A_{1}\left(x_{m}, y_{m}\right)(t): m=1,2,3, \ldots\right\}$, and $\left(A_{1} V\right)^{\prime}(t)=\left\{A_{1}^{\prime}\left(x_{m}, y_{m}\right)(t): m=\right.$ $1,2,3, \ldots\}$.

By (2.21), we know that the infinite integral $\int_{0}^{+\infty}\left\|f\left(t, x(t), x^{\prime}(t), y(t), y^{\prime}(t)\right)\right\| \mathrm{d} t$ is convergent uniformly for $m=1,2,3, \ldots$. So, for any $\epsilon>0$, we can choose a sufficiently large $T>0$ such that

$$
\int_{T}^{+\infty}\left\|f\left(t, x(t), x^{\prime}(t), y(t), y^{\prime}(t)\right)\right\| \mathrm{d} t<\epsilon
$$

Then, by [1, Theorem 1.2.3], (2.44), (3.1), (3.3), $\left(\mathrm{H}_{2}\right)$, and Lemma 2.7, we obtain

$$
\begin{aligned}
\alpha\left(\frac{\left(A_{1} V\right)(t)}{1+t}\right) \leq & 2 \frac{D_{0}}{1+t} \int_{0}^{T} \alpha\left(\left\{f\left(s, x_{m}(s), x_{m}^{\prime}(s), y_{m}(s), y_{m}^{\prime}(s)\right):\left(x_{m}, y_{m}\right) \in V\right\}\right) \mathrm{d} s+2 \epsilon \\
& +2 \int_{0}^{T} \frac{t}{1+t} \alpha\left(\left\{f\left(s, x_{m}(s), x_{m}^{\prime}(s), y_{m}(s), y_{m}^{\prime}(s)\right):\left(x_{m}, y_{m}\right) \in V\right\}\right) \mathrm{d} s+2 \epsilon \\
\leq & 2 D_{0} \int_{0}^{T} \alpha\left(\left\{f\left(s, x_{m}(s), x_{m}^{\prime}(s), y_{m}(s), y_{m}^{\prime}(s)\right):\left(x_{m}, y_{m}\right) \in V\right\}\right) \mathrm{d} s \\
& +2 \int_{0}^{T} \alpha\left(\left\{f\left(s, x_{m}(s), x_{m}^{\prime}(s), y_{m}(s), y_{m}^{\prime}(s)\right):\left(x_{m}, y_{m}\right) \in V\right\}\right) \mathrm{d} s+4 \epsilon \\
\leq & \left(2 D_{0}+2\right) \int_{0}^{+\infty} \alpha\left(\left\{f\left(s, x_{m}(s), x_{m}^{\prime}(s), y_{m}(s), y_{m}^{\prime}(s)\right):\left(x_{m}, y_{m}\right) \in V\right\}\right) \mathrm{d} s+4 \epsilon \\
\leq & \left(2 D_{0}+2\right) \alpha_{X}(V) \int_{0}^{+\infty}\left(L_{00}(s)+K_{00}(s)\right)(1+s)+\left(L_{01}(s)+K_{01}(s)\right) \mathrm{d} s+4 \epsilon,
\end{aligned}
$$




$$
\begin{aligned}
\alpha\left(\left(A_{1}^{\prime} V\right)(t)\right) & \leq 2 \int_{0}^{+\infty} \alpha\left(\left\{f\left(s, x_{m}(s), x_{m}^{\prime}(s), y_{m}(s), y_{m}^{\prime}(s)\right):\left(x_{m}, y_{m}\right) \in V\right\}\right) \mathrm{d} s+2 \epsilon \\
& \leq 2 \alpha_{X}(V) \int_{0}^{+\infty}\left(L_{00}(s)+K_{00}(s)\right)(1+s)+\left(L_{01}(s)+K_{01}(s)\right) \mathrm{d} s+2 \epsilon
\end{aligned}
$$

It follows from (3.2) and (3.4) that

$$
\alpha_{D}\left(A_{1} V\right) \leq\left(2 D_{0}+2\right) \alpha_{X}(V) \int_{0}^{+\infty}\left(L_{00}(s)+K_{00}(s)\right)(1+s)+\left(L_{01}(s)+K_{01}(s)\right) \mathrm{d} s .
$$

In the same way, we get

$$
\alpha_{D}\left(A_{2} V\right) \leq\left(2 D_{1}+2\right) \alpha_{X}(V) \int_{0}^{+\infty}\left(L_{10}(s)+K_{10}(s)\right)(1+s)+\left(L_{11}(s)+K_{11}(s)\right) \mathrm{d} s .
$$

On the other hand, $\alpha_{X}(V) \leq \alpha_{X}\{\overline{\mathrm{CO}}(\{u\} \cup(A V))\}=\alpha_{X}(A V)$. Then, (3.5), (3.6), $\left(\mathrm{H}_{2}\right)$, and Lemma 2.7 imply $\alpha_{X}(V)=0$, that is, $V$ is relatively compact in $D C^{1}[J, E] \times D C^{1}[J, E]$. Hence, the Mönch fixed point theorem guarantees that $A$ has a fixed point $(\bar{x}, \bar{y})$ in $Q^{*}$. Thus, Theorem 3.1 is proved.

Theorem 3.2. Let cone $P$ be normal and conditions $\left(H_{1}\right)-\left(H_{3}\right)$ be satisfied. Then BVP (1.2) has a positive solution $(\bar{x}, \bar{y}) \in Q \cap\left(C^{2}\left[J_{+}^{\prime}, E\right] \times C^{2}\left[J_{+}^{\prime}, E\right]\right)$ which is minimal in the sense that $u^{(i)}(t) \geq$ $\bar{x}^{(i)}(t), v^{(i)}(t) \geq \bar{y}^{(i)}(t), t \in J(i=0,1)$ for any positive solution $(u, v) \in Q \cap\left(C^{2}\left[J_{+}^{\prime}, E\right] \times C^{2}\left[J_{+}^{\prime}, E\right]\right)$ of $B V P(1.2)$. Moreover, $\|((\bar{x}, \bar{y}))\|_{X} \leq 2 \gamma+\left\|\left(u_{0}, v_{0}\right)\right\|_{X}$, and there exists a monotone iterative sequence $\left\{\left(u_{n}(t), v_{n}(t)\right)\right\}$ such that $u_{n}^{(i)}(t) \rightarrow \bar{x}^{(i)}(t), v_{n}^{(i)}(t) \rightarrow \bar{y}^{(i)}(t)$ as $n \rightarrow \infty(i=0,1)$ uniformly on $J$ and $u_{n}^{\prime \prime}(t) \rightarrow \bar{x}^{\prime \prime}(t), v_{n}^{\prime \prime}(t) \rightarrow \bar{y}^{\prime \prime}(t)$ as $n \rightarrow \infty$ for any $t \in J_{+}$, where

$$
\begin{aligned}
u_{0}(t)= & \frac{1}{1-\sum_{i=1}^{m-2} \alpha_{i}}\left[\left(\sum_{i=1}^{m-2} \alpha_{i} \xi_{i}\right) x_{\infty}+\sum_{i=1}^{m-2} \alpha_{i} \int_{0}^{\xi_{i}} \int_{s}^{+\infty} f\left(\tau, x_{0}^{*}, x_{0}^{*}, y_{0}^{*}, y_{0}^{*}\right) \mathrm{d} \tau \mathrm{d} s\right] \\
& +\int_{0}^{t} \int_{s}^{+\infty} f\left(\tau, x_{0}^{*}, x_{0}^{*}, y_{0}^{*}, y_{0}^{*}\right) \mathrm{d} \tau \mathrm{d} s+t x_{\infty}, \\
v_{0}(t)= & \frac{1}{1-\sum_{i=1}^{m-2} \beta_{i}}\left[\left(\sum_{i=1}^{m-2} \beta_{i} \xi_{i}\right) y_{\infty}+\sum_{i=1}^{m-2} \beta_{i} \int_{0}^{\xi_{i}} \int_{s}^{+\infty} g\left(\tau, x_{0}^{*}, x_{0}^{*}, y_{0}^{*}, y_{0}^{*}\right) \mathrm{d} \tau \mathrm{d} s\right] \\
& +\int_{0}^{t} \int_{s}^{+\infty} g\left(\tau, x_{0}^{*}, x_{0}^{*}, y_{0}^{*}, y_{0}^{*}\right) \mathrm{d} \tau \mathrm{d} s+t y_{\infty},
\end{aligned}
$$




$$
\begin{aligned}
u_{n}(t)= & \frac{1}{1-\sum_{i=1}^{m-2} \alpha_{i}} \\
& \times\left[\left(\sum_{i=1}^{m-2} \alpha_{i} \xi_{i}\right) x_{\infty}+\sum_{i=1}^{m-2} \alpha_{i} \int_{0}^{\xi_{i}} \int_{s}^{+\infty} f\left(\tau, u_{n-1}(\tau), u_{n-1}^{\prime}(\tau), v_{n-1}(\tau), v_{n-1}^{\prime}(\tau)\right) \mathrm{d} \tau \mathrm{d} s\right] \\
& +\int_{0}^{t} \int_{s}^{+\infty} f\left(\tau, u_{n-1}(\tau), u_{n-1}^{\prime}(\tau), v_{n-1}(\tau), v_{n-1}^{\prime}(\tau)\right) \mathrm{d} \tau \mathrm{d} s+t x_{\infty}, \quad \forall t \in J(n=1,2,3, \ldots), \\
v_{n}(t)= & \frac{1}{1-\sum_{i=1}^{m-2} \beta_{i}} \\
& \times\left[\left(\sum_{i=1}^{m-2} \beta_{i} \xi_{i}\right) y_{\infty}+\sum_{i=1}^{m-2} \beta_{i} \int_{0}^{\xi_{i}} \int_{s}^{+\infty} g\left(\tau, u_{n-1}(\tau), u_{n-1}^{\prime}(\tau), v_{n-1}(\tau), v_{n-1}^{\prime}(\tau)\right) \mathrm{d} \tau \mathrm{d} s\right] \\
& +\int_{0}^{t} \int_{s}^{+\infty} g\left(\tau, u_{n-1}(\tau), u_{n-1}^{\prime}(\tau), v_{n-1}(\tau), v_{n-1}^{\prime}(\tau)\right) \mathrm{d} \tau \mathrm{d} s+t y_{\infty}, \quad \forall t \in J(n=1,2,3, \ldots) .
\end{aligned}
$$

Proof. From (3.7), one can see that $\left(u_{0}, v_{0}\right) \in C[J, E] \times C[J, E]$ and

$$
u_{0}^{\prime}(t)=\int_{t}^{+\infty} f\left(s, x_{0}^{*}, x_{0}^{*}, y_{0}^{*}, y_{0}^{*}\right) \mathrm{d} s+x_{\infty}
$$

By (3.7) and (3.11), we have that $u_{0}^{(i)} \geq x_{\infty} \geq x_{0}^{*}(i=0,1)$ and

$$
\begin{aligned}
\left\|u_{0}(t)\right\| \leq & \int_{0}^{t} \int_{s}^{+\infty}\left\|f\left(\tau, x_{0}^{*}, x_{0}^{*}, y_{0}^{*}, y_{0}^{*}\right)\right\| \mathrm{d} \tau \mathrm{d} s+t\left\|x_{\infty}\right\|+\frac{\sum_{i=1}^{m-2} \alpha_{i} \xi_{i}}{1-\sum_{i=1}^{m-2} \alpha_{i}}\left\|x_{\infty}\right\| \\
& +\frac{1}{1-\sum_{i=1}^{m-2} \alpha_{i}} \sum_{i=1}^{m-2} \alpha_{i} \int_{0}^{\xi_{m-2}} \int_{s}^{+\infty}\left\|f\left(\tau, x_{0}^{*}, x_{0}^{*}, y_{0}^{*}, y_{0}^{*}\right)\right\| \mathrm{d} \tau \mathrm{d} s \\
\leq & t\left(\int_{0}^{+\infty}\left\|f\left(\tau, x_{0}^{*}, x_{0}^{*}, y_{0}^{*}, y_{0}^{*}\right)\right\| \mathrm{d} \tau+\left\|x_{\infty}\right\|\right)+\frac{\sum_{i=1}^{m-2} \alpha_{i} \xi_{i}}{1-\sum_{i=1}^{m-2} \alpha_{i}}\left\|x_{\infty}\right\| \\
& +\frac{1}{1-\sum_{i=1}^{m-2} \alpha_{i}} \sum_{i=1}^{m-2} \alpha_{i} \xi_{m-2}\left(\int_{0}^{+\infty}\left\|f\left(\tau, x_{0}^{*}, x_{0}^{*}, y_{0}^{*}, y_{0}^{*}\right)\right\| \mathrm{d} \tau\right) \\
\leq & t\left[\int_{0}^{+\infty} a_{0}(s)+b_{0}(s) h_{0}\left(\left\|x_{0}^{*}\right\|,\left\|x_{0}^{*}\right\|,\left\|y_{0}^{*}\right\|,\left\|y_{0}^{*}\right\|\right) \mathrm{d} s+\left\|x_{\infty}\right\|\right]+\frac{\sum_{i=1}^{m-2} \alpha_{i} \xi_{i}}{1-\sum_{i=1}^{m-2} \alpha_{i}}\left\|x_{\infty}\right\| \\
& +\frac{1}{1-\sum_{i=1}^{m-2} \alpha_{i}} \sum_{i=1}^{m-2} \alpha_{i} \xi_{m-2}\left(\int_{0}^{+\infty} a_{0}(s)+b_{0}(s) h_{0}\left(\left\|x_{0}^{*}\right\|,\left\|x_{0}^{*}\right\|,\left\|y_{0}^{*}\right\|,\left\|y_{0}^{*}\right\|\right) \mathrm{d} s\right),
\end{aligned}
$$




$$
\begin{aligned}
\left\|u_{0}^{\prime}(t)\right\| & \leq \int_{t}^{+\infty}\left\|f\left(\tau, x_{0}^{*}, x_{0}^{*}, y_{0}^{*}, y_{0}^{*}\right)\right\| \mathrm{d} \tau+\left\|x_{\infty}\right\| \\
& \leq \int_{0}^{+\infty} a_{0}(s)+b_{0}(s) h_{0}\left(\left\|x_{0}^{*}\right\|,\left\|x_{0}^{*}\right\|,\left\|y_{0}^{*}\right\|,\left\|y_{0}^{*}\right\|\right) \mathrm{d} s+\left\|x_{\infty}\right\|,
\end{aligned}
$$

which imply that $\left\|u_{0}\right\|_{D}<\infty$. Similarly, we have $\left\|v_{0}\right\|_{D}<\infty$. Thus, $\left(u_{0}, v_{0}\right) \in D C^{1}[J, E] \times$ $D C^{1}[J, E]$. It follows from (2.13) and (3.9) that

$$
\left(u_{n}, v_{n}\right)(t)=A\left(u_{n-1}, v_{n-1}\right)(t), \quad \forall t \in J, n=1,2,3, \ldots
$$

By Lemma 2.1, we get $\left(u_{n}, v_{n}\right) \in Q$ and

$$
\left\|\left(u_{n}, v_{n}\right)\right\|_{X}=\left\|A\left(u_{n-1}, v_{n-1}\right)\right\|_{X} \leq \frac{1}{2}\left\|\left(u_{n-1}, v_{n-1}\right)\right\|_{X}+\gamma .
$$

By Lemma 2.6 and (3.13), we have

$$
\left(x_{0}^{*}, y_{0}^{*}\right) \leq\left(u_{0}^{(i)}(t), v_{0}^{(i)}(t)\right) \leq\left(u_{1}^{(i)}(t), v_{1}^{(i)}(t)\right) \leq \cdots \leq\left(u_{n}^{(i)}(t), v_{n}^{(i)}(t)\right) \leq \cdots, \quad \forall t \in J(i=0,1) .
$$

It follows from (3.14), by induction, that

$$
\begin{aligned}
\left\|\left(u_{n}, v_{n}\right)\right\|_{X} & \leq \gamma+\left(\frac{1}{2}\right) \gamma+\cdots+\left(\frac{1}{2}\right)^{n-1} \gamma+\left(\frac{1}{2}\right)^{n}\left\|\left(u_{0}, v_{0}\right)\right\|_{X} \\
& \leq \frac{\gamma\left(1-(1 / 2)^{n}\right)}{1-1 / 2}+\left\|\left(u_{0}, v_{0}\right)\right\|_{X} \\
& \leq 2 \gamma+\left\|\left(u_{0}, v_{0}\right)\right\|_{X} \quad(n=1,2,3, \ldots) .
\end{aligned}
$$

Let $K=\left\{(x, y) \in Q:\|(x, y)\|_{X} \leq 2 \gamma+\left\|\left(u_{0}, v_{0}\right)\right\|_{X}\right\}$. Then, $K$ is a bounded closed convex set in space $D C^{1}[J, E] \times D C^{1}[J, E]$ and operator $A$ maps $K$ into $K$. Clearly, $K$ is not empty since $\left(u_{0}, v_{0}\right) \in K$. Let $W=\left\{\left(u_{n}, v_{n}\right): n=0,1,2, \ldots\right\}, A W=\left\{A\left(u_{n}, v_{n}\right): n=0,1,2, \ldots\right\}$. Obviously, $W \subset K$ and $W=\left\{\left(u_{0}, v_{0}\right)\right\} \cup A(W)$. Similar to above proof of Theorem 3.1, we can obtain $\alpha_{X}(A W)=0$, that is, $W$ is relatively compact in $D C^{1}[J, E] \times D C^{1}[J, E]$. So, there exists an $(\bar{x}, \bar{y}) \in D C^{1}[J, E] \times D C^{1}[J, E]$ and a subsequence $\left\{\left(u_{n_{j}}, v_{n_{j}}\right): j=1,2,3, \ldots\right\} \subset W$ such that $\left\{\left(u_{n_{i}}, v_{n_{j}}\right)(t): j=1,2,3, \ldots\right\}$ converges to $\left(\bar{x}^{(i)}(t), \bar{y}^{(i)}(t)\right)$ uniformly on $J(i=0,1)$. Since that $P$ is normal and $\left\{\left(u_{n}^{(i)}(t), v_{n}^{(i)}(t)\right): n=1,2,3, \ldots\right\}$ is nondecreasing, it is easy to see that the entire sequence $\left\{\left(u_{n}^{(i)}(t), v_{n}^{(i)}(t)\right): n=1,2,3, \ldots\right\}$ converges to $\left(\bar{x}^{(i)}(t), \bar{y}^{(i)}(t)\right)$ uniformly on $J(i=0,1)$. Since $\left(u_{n}, v_{n}\right) \in K$ and $K$ are closed convex sets in space $D C^{1}[J, E] \times D C^{1}[J, E]$, we have $(\bar{x}, \bar{y}) \in K$. It is clear,

$$
f\left(s, u_{n}(s), u_{n}^{\prime}(s), v_{n}(s), v_{n}^{\prime}(s)\right) \longrightarrow f\left(s, \bar{x}(s), \bar{x}^{\prime}(s), \bar{y}(s), \bar{y}^{\prime}(s)\right), \quad \text { as } n \longrightarrow \infty, \forall s \in J_{+} .
$$


By $\left(\mathrm{H}_{1}\right)$ and (3.16), we have

$$
\begin{aligned}
\| f(s & \left., u_{n}(s), u_{n}^{\prime}(s), v_{n}(s), v_{n}^{\prime}(s)\right)-f\left(s, \bar{x}(s), \bar{x}^{\prime}(s), \bar{y}(s), \bar{y}^{\prime}(s)\right) \| \\
& \leq 8 \epsilon_{0} c(s)(1+s)\left\|\left(u_{n}, v_{n}\right)\right\|_{X}+2 a_{0}(s)+2 M_{0} b_{0}(s) \\
& \leq 8 \epsilon_{0} c(s)(1+s)\left(2 \gamma+\left\|\left(u_{0}, v_{0}\right)\right\|_{X}\right)+2 a_{0}(s)+2 M_{0} b_{0}(s) .
\end{aligned}
$$

Noticing (3.17) and (3.18) and taking limit as $n \rightarrow \infty$ in (3.9), we obtain

$$
\begin{aligned}
\bar{x}(t)= & \frac{1}{1-\sum_{i=1}^{m-2} \alpha_{i}}\left[\left(\sum_{i=1}^{m-2} \alpha_{i} \xi_{i}\right) x_{\infty}+\sum_{i=1}^{m-2} \alpha_{i} \int_{0}^{\xi_{i}} \int_{s}^{+\infty} f\left(\tau, \bar{x}(\tau), \bar{x}^{\prime}(\tau), \bar{y}(\tau), \bar{y}^{\prime}(\tau)\right) \mathrm{d} \tau \mathrm{d} s\right] \\
& +\int_{0}^{t} \int_{s}^{+\infty} f\left(\tau, \bar{x}(\tau), \bar{x}^{\prime}(\tau), \bar{y}(\tau), \bar{y}^{\prime}(\tau)\right) \mathrm{d} \tau \mathrm{d} s+t x_{\infty} .
\end{aligned}
$$

In the same way, taking limit as $n \rightarrow \infty$ in (3.10), we get

$$
\begin{aligned}
\bar{y}(t)= & \frac{1}{1-\sum_{i=1}^{m-2} \beta_{i}}\left[\left(\sum_{i=1}^{m-2} \beta_{i} \xi_{i}\right) y_{\infty}+\sum_{i=1}^{m-2} \beta_{i} \int_{0}^{\xi_{i}} \int_{s}^{+\infty} g\left(\tau, \bar{x}(\tau), \bar{x}^{\prime}(\tau), \bar{y}(\tau), \bar{y}^{\prime}(\tau)\right) \mathrm{d} \tau \mathrm{d} s\right] \\
& +\int_{0}^{t} \int_{s}^{+\infty} g\left(\tau, \bar{x}(\tau), \bar{x}^{\prime}(\tau), \bar{y}(\tau), \bar{y}^{\prime}(\tau)\right) \mathrm{d} \tau \mathrm{d} s+t y_{\infty},
\end{aligned}
$$

which together with (3.19) and Lemma 2.2 implies that $(\bar{x}, \bar{y}) \in K \cap C^{2}\left[J_{+}, E\right] \times C^{2}\left[J_{+}, E\right]$ and $(\bar{x}(t), \bar{y}(t))$ is a positive solution of BVP (1.2). Differentiating (3.9) twice, we get

$$
u_{n}^{\prime \prime}(t)=-f\left(t, u_{n-1}(t), u_{n-1}^{\prime}(t), v_{n-1}(t), v_{n-1}^{\prime}(t)\right), \quad \forall t \in J_{+}^{\prime}, n=1,2,3, \ldots
$$

Hence, by (3.17), we obtain

$$
\lim _{n \rightarrow \infty} u_{n}^{\prime \prime}(t)=-f\left(t, \bar{x}(t), \bar{x}^{\prime}(t), \bar{y}(t), \bar{y}^{\prime}(t)\right)=\bar{x}^{\prime \prime}(t), \quad \forall t \in J_{+}^{\prime}
$$

Similarly, we have

$$
\lim _{n \rightarrow \infty} v_{n}^{\prime \prime}(t)=-g\left(t, \bar{x}(t), \bar{x}^{\prime}(t), \bar{y}(t), \bar{y}^{\prime}(t)\right)=\bar{y}^{\prime \prime}(t), \quad \forall t \in J_{+}^{\prime}
$$

Let $(p(t), q(t))$ be any positive solution of BVP (1.2). By Lemma 2.2, we have $(p, q) \in Q$ and $(p(t), q(t))=(A(p, q))(t)$, for $t \in J$. It is clear that $p^{(i)}(t) \geq x_{0}^{*}>\theta, q^{(i)}(t) \geq y_{0}^{*}>\theta$ for any 
$t \in J(i=0,1)$. So, by Lemma 2.6, we have $p^{(i)}(t) \geq u_{0}^{(i)}(t), q^{(i)}(t) \geq v_{0}^{(i)}(t)$ for any $t \in J(i=$ $0,1)$. Assume that $p^{(i)}(t) \geq u_{n-1}^{(i)}(t), q^{(i)}(t) \geq v_{n-1}^{(i)}(t)$ for $t \in J, n \geq 1(i=0,1)$. Then, it follows from Lemma 2.6 that $\left.\left.\left(A_{1}^{(i)}(p, q)(t), A_{2}^{(i)}(p, q)(t)\right) \geq\left(A_{1}^{(i)}\left(u_{n-1}, v_{n-1}\right)\right)(t), A_{2}^{(i)}\left(u_{n-1}, v_{n-1}\right)\right)(t)\right)$ for $t \in J(i=0,1)$, that is, $\left(p^{(i)}(t), q^{(i)}(t)\right) \geq\left(u_{n}^{(i)}(t), v_{n}^{(i)}(t)\right)$ for $t \in J(i=0,1)$. Hence, by induction, we get

$$
p^{(i)}(t) \geq \bar{x}_{n}^{(i)}(t), \quad q^{(i)}(t) \geq \bar{y}_{n}^{(i)}(t) \quad \forall t \in J(i=0,1 ; m=0,1,2, \ldots) .
$$

Now, taking limits in (3.24), we get $p^{(i)}(t) \geq \bar{x}^{(i)}(t), q^{(i)}(t) \geq \bar{y}^{(i)}(t)$ for $t \in J(i=0,1)$, and the theorem is proved.

Theorem 3.3. Let cone $P$ be fully regular and conditions $\left(H_{1}\right)$ and $\left(H_{3}\right)$ be satisfied. Then the conclusion of Theorem 3.2 holds.

Proof. The proof is almost the same as that of Theorem 3.2. The only difference is that, instead of using condition $\left(\mathrm{H}_{2}\right)$, the conclusion $\alpha_{X}(W)=0$ is implied directly by (3.15) and (3.16), the full regularity of $P$ and Lemma 2.4 .

\section{An Example}

Consider the infinite system of scalar singular second order three-point boundary value problems:

$$
\begin{aligned}
-x_{n}^{\prime \prime}(t) & =\frac{1}{3 n^{2} \sqrt{t}(1+t)}\left(2+x_{n}(t)+y_{n}(t)+x_{2 n}^{\prime}(t)+y_{3 n}^{\prime}(t)+\frac{1}{2 n^{2} x_{n}(t)}+\frac{1}{8 n^{3} x_{2 n}^{\prime}(t)}\right)^{1 / 3} \\
& +\frac{1}{3 e^{2 t}(1+t)} \ln \left(1+x_{n}(t)\right), \\
-y_{n}^{\prime \prime}(t) & =\frac{1}{6 n^{3} \sqrt{t^{2}}(1+t)}\left(1+x_{3 n}(t)+x_{4 n}^{\prime}(t)+\frac{1}{3 n^{2} y_{3 n}(t)}+\frac{1}{4 n^{3} y_{2 n}^{\prime}(t)}\right)^{1 / 5} \\
& +\frac{1}{6 e^{3 t^{2}}(1+t)} \ln \left(1+y_{2 n}^{\prime}(t)\right), \\
x_{n}(0) & =\frac{2}{3} x_{n}(1), \quad x_{n}^{\prime}(\infty)=\frac{1}{n}, \quad y_{n}(0)=\frac{3}{4} y_{n}(1), \quad y_{n}^{\prime}(\infty)=\frac{1}{2 n} \quad(n=1,2, \ldots) .
\end{aligned}
$$

Proposition 4.1. Infinite system (4.1) has a minimal positive solution $\left(x_{n}(t), y_{n}(t)\right)$ satisfying $x_{n}(t), x_{n}^{\prime}(t) \geq 1 / n, y_{n}(t), y_{n}^{\prime}(t) \geq 1 / 2 n$ for $0 \leq t<+\infty(n=1,2,3, \ldots)$.

Proof. Let $E=c_{0}=\left\{x=\left(x_{1}, \ldots, x_{n}, \ldots\right): x_{n} \rightarrow 0\right\}$ with the norm $\|x\|=\sup _{n}\left|x_{n}\right|$. Obviously, $(E,\|\cdot\|)$ is a real Banach space. Choose $P=\left\{x=\left(x_{n}\right) \in c_{0}: x_{n} \geq 0, n=1,2,3, \ldots\right\}$. It is easy to verify that $P$ is a normal cone in $E$ with normal constants 1 . Now we consider infinite 
system (4.1), which can be regarded as a BVP of form (1.2) in $E$ with $\alpha_{1}=2 / 3, \beta_{1}=3 / 4, \xi_{1}=$ $1, x_{\infty}=(1,1 / 2,1 / 3, \ldots), y_{\infty}=(1 / 2,1 / 4,1 / 6, \ldots)$. In this situation, $x=\left(x_{1}, \ldots, x_{n}, \ldots\right), u=$ $\left(u_{1}, \ldots, u_{n}, \ldots\right), y=\left(y_{1}, \ldots, y_{n}, \ldots\right), v=\left(v_{1}, \ldots, v_{n}, \ldots\right), f=\left(f_{1}, \ldots, f_{n}, \ldots\right)$, in which

$$
\begin{aligned}
f_{n}(t, x, u, y, v)= & \frac{1}{3 n^{2} \sqrt{t}(1+t)}\left(2+x_{n}+y_{n}+u_{2 n}+v_{3 n}+\frac{1}{2 n^{2} x_{n}}+\frac{1}{8 n^{3} u_{2 n}}\right)^{1 / 3} \\
& +\frac{1}{3 e^{2 t}(1+t)} \ln \left(1+x_{n}\right), \\
g_{n}(t, x, u, y, v)= & \frac{1}{6 n^{3} \sqrt[3]{t^{2}}(1+t)}\left(1+x_{3 n}+u_{4 n}+\frac{1}{3 n^{2} y_{3 n}}+\frac{1}{4 n^{3} v_{2 n}}\right)^{1 / 5} \\
& +\frac{1}{6 e^{3 t}(1+t)} \ln \left(1+v_{2 n}\right) .
\end{aligned}
$$

Let $x_{0}^{*}=x_{\infty}=(1,1 / 2,1 / 3, \ldots), y_{0}^{*}=y_{\infty}=(1 / 2,1 / 4,1 / 6, \ldots)$. Then $P_{0 \lambda}=\{x=$ $\left.\left(x_{1}, x_{2}, \ldots, x_{n}, \ldots\right): x_{n} \geq \lambda / n, n=1,2,3, \ldots\right\}, P_{1 \lambda}=\left\{y=\left(y_{1}, y_{2}, \ldots, y_{n}, \ldots\right): y_{n} \geq \lambda / 2 n, n=\right.$ $1,2,3, \ldots\}$, for $\lambda>0$. It is clear, $f, g \in C\left[J_{+} \times P_{0 \lambda} \times P_{0 \lambda} \times P_{1 \lambda} \times P_{1 \lambda}, P\right]$ for any $\lambda>0$. Notice that $e^{3 t}>\sqrt[3]{t^{2}}, e^{2 t}>\sqrt{t}$ for $t>0$, by (4.2), we get

$$
\begin{gathered}
\|f(t, x, u, y, v)\| \leq \frac{1}{3 \sqrt{t}}\left[\left(\frac{11}{4}+\|x\|+\|u\|+\|v\|+\|y\|\right)^{1 / 3}+\ln (1+\|x\|)\right], \\
\|g(t, x, u, y, v)\| \leq \frac{1}{6 \sqrt[3]{t^{2}}}\left[(4+\|x\|+\|u\|)^{1 / 5}+\ln (1+\|v\|)\right],
\end{gathered}
$$

which imply $\left(\mathrm{H}_{1}\right)$ is satisfied for $a_{0}(t)=0, b_{0}(t)=c_{0}(t)=1 / 3 \sqrt{t}, a_{1}(t)=0, b_{1}(t)=c_{1}(t)=$ $1 / 6 \sqrt[3]{t^{2}}$ and

$$
\begin{gathered}
h_{0}\left(u_{0}, u_{1}, u_{2}, u_{3}\right)=\left(\frac{11}{4}+u_{0}+u_{1}+u_{2}+u_{3}\right)^{1 / 3}+\ln \left(1+u_{0}\right), \\
h_{1}\left(u_{0}, u_{1}, u_{2}, u_{3}\right)=\left(4+u_{0}+u_{1}\right)^{1 / 5}+\ln \left(1+u_{3}\right) .
\end{gathered}
$$

Let $f^{1}=\left\{f_{1}^{1}, f_{2}^{1}, \ldots, f_{n}^{1}, \ldots\right\}, f^{2}=\left\{f_{1}^{2}, f_{2}^{2}, \ldots, f_{n}^{2}, \ldots\right\}$, and $g^{1}=\left\{g_{1}^{1}, g_{2}^{1}, \ldots, g_{n}^{1}, \ldots\right\}, g^{2}=$ $\left\{g_{1}^{2}, g_{2}^{2}, \ldots, g_{n}^{2}, \ldots\right\}$, where

$$
\begin{gathered}
f_{n}^{1}(t, x, u, y, v)=\frac{1}{3 n^{2} \sqrt{t}(1+t)}\left(2+x_{n}+y_{n}+u_{2 n}+v_{3 n}+\frac{1}{2 n^{2} x_{n}}+\frac{1}{8 n^{3} u_{2 n}}\right)^{1 / 3}, \\
f_{n}^{2}(t, x, u, y, v)=\frac{1}{3 e^{2 t}(1+t)} \ln \left(1+x_{n}\right), \\
g_{n}^{1}(t, x, u, y, v)=\frac{1}{6 n^{3} \sqrt[3]{t^{2}}(1+t)}\left(1+x_{3 n}+u_{4 n}+\frac{1}{3 n^{2} y_{3 n}}+\frac{1}{4 n^{3} v_{2 n}}\right)^{1 / 5}, \\
g_{n}^{2}(t, x, u, y, v)=\frac{1}{6 e^{3 t}(1+t)} \ln \left(1+v_{2 n}\right) .
\end{gathered}
$$


Let $t \in J_{+}, R>0$ be given, and $\left\{z^{(m)}\right\}$ be any sequence in $f^{1}\left(t, P_{0 R^{\prime}}^{*}, P_{0 R^{\prime}}^{*} P_{1 R^{\prime}}^{*} P_{1 R}^{*}\right)$, where $z^{(m)}=$ $\left(z_{1}^{(m)}, \ldots, z_{n}^{(m)}, \ldots\right)$. By (4.5), we have

$$
0 \leq z_{n}^{(m)} \leq \frac{1}{3 n^{2} \sqrt{t}}\left(\frac{11}{4}+4 R\right)^{1 / 3} \quad(n, m=1,2,3, \ldots)
$$

So, $\left\{z_{n}^{(m)}\right\}$ is bounded and by the diagonal method together with the method of constructing subsequence, we can choose a subsequence $\left\{m_{i}\right\} \subset\{m\}$ such that

$$
\left\{z_{n}^{(m)}\right\} \longrightarrow \bar{z}_{n} \quad \text { as } i \longrightarrow \infty(n=1,2,3, \ldots)
$$

which implies by virtue of (4.9)

$$
0 \leq \bar{z}_{n} \leq \frac{1}{3 n^{2} \sqrt{t}}\left(\frac{11}{4}+4 R\right)^{1 / 3} \quad(n=1,2,3, \ldots) .
$$

Hence $\bar{z}=\left(\bar{z}_{1}, \ldots, \bar{z}_{n}, \ldots\right) \in c_{0}$. It is easy to see from (4.9)-(4.11) that

$$
\left\|z^{\left(m_{i}\right)}-\bar{z}\right\|=\sup _{n}\left|z_{n}^{\left(m_{i}\right)}-\bar{z}_{n}\right| \longrightarrow 0 \quad \text { as } i \longrightarrow \infty .
$$

Thus, we have proved that $f^{1}\left(t, P_{0 R}^{*}, P_{0 R}^{*}, P_{1 R}^{*}, P_{1 R}^{*}\right)$ is relatively compact in $c_{0}$.

For any $t \in J_{+}, R>0, x, y, \bar{x}, \bar{y} \in D \subset P_{0 R^{\prime}}^{*}$ we have by (4.6)

$$
\begin{aligned}
\left|f_{n}^{2}(t, x, u, y, v)-f_{n}^{2}(t, \bar{x}, \bar{u}, \bar{y}, \bar{v})\right| & =\frac{1}{3 e^{2 t}(1+t)}\left|\ln \left(1+x_{n}\right)-\ln \left(1+\bar{x}_{n}\right)\right| \\
& \leq \frac{1}{3 e^{2 t}(1+t)} \frac{\left|x_{n}-\bar{x}_{n}\right|}{1+\xi_{n}},
\end{aligned}
$$

where $\xi_{n}$ is between $x_{n}$ and $\bar{x}_{n}$. By (4.13), we get

$$
\left\|f^{2}(t, x, u, y, v)-f^{2}(t, \bar{x}, \bar{u}, \bar{y}, \bar{v})\right\| \leq \frac{1}{3 e^{2 t}(1+t)}\|x-\bar{x}\|, \quad x, y, \bar{x}, \bar{y} \in D
$$

In the same way, we can prove that $g^{1}\left(t, P_{0 R^{\prime}}^{*}, P_{0 R^{\prime}}^{*} P_{1 R^{\prime}}^{*}, P_{1 R}^{*}\right)$ is relatively compact in $c_{0}$, and we can also get

$$
\left\|g^{2}(t, x, u, y, v)-g^{2}(t, \bar{x}, \bar{u}, \bar{y}, \bar{v})\right\| \leq \frac{1}{6 e^{3 t}(1+t)}\|v-\bar{v}\|, \quad x, y, \bar{x}, \bar{y} \in D
$$

Thus, by (4.14) and (4.15), it is easy to see that $\left(\mathrm{H}_{2}\right)$ holds for $L_{00}(t)=1 / 3 e^{2 t}(1+t), K_{11}(t)=$ $1 / 6 e^{3 t}(1+t)$. Thus, our conclusion follows from Theorem 3.1. This completes the proof. 


\section{Acknowledgment}

The project is supported financially by the National Natural Science Foundation of China (10671167) and the Natural Science Foundation of Liaocheng University (31805).

\section{References}

[1] D. J. Guo, V. Lakshmikantham, and X. Z. Liu, Nonlinear Integral Equations in Abstract Spaces, vol. 373 of Mathematics and Its Applications, Kluwer Academic Publishers, Dordrecht, The Netherlands, 1996.

[2] K. Deimling, Ordinary Differential Equations in Banach Spaces, vol. 596 of Lecture Notes in Mathematics, Springer, Berlin, Germany, 1977.

[3] V. Lakshmikantham and S. Leela, Nonlinear Differential Equations in Abstract Spaces, vol. 2 of International Series in Nonlinear Mathematics: Theory, Methods and Applications, Pergamon Press, Oxford, UK, 1981.

[4] D. J. Guo and V. Lakshmikantham, Nonlinear Problems in Abstract Cones, vol. 5 of Notes and Reports in Mathematics in Science and Engineering, Academic Press, Boston, Mass, USA, 1988.

[5] Y. Liu, "Boundary value problems for second order differential equations on unbounded domains in a Banach space," Applied Mathematics and Computation, vol. 135, no. 2-3, pp. 569-583, 2003.

[6] C. P. Gupta, "Solvability of a three-point nonlinear boundary value problem for a second order ordinary differential equation," Journal of Mathematical Analysis and Applications, vol. 168, no. 2, pp. 540-551, 1992.

[7] W. Feng and J. R. L. Webb, "Solvability of $m$-point boundary value problems with nonlinear growth," Journal of Mathematical Analysis and Applications, vol. 212, no. 2, pp. 467-480, 1997.

[8] J. X. Sun, X. Xu, and D. O’Regan, "Nodal solutions for $m$-point boundary value problems using bifurcation methods," Nonlinear Analysis: Theory, Methods E Applications, vol. 68, no. 10, pp. 30343046, 2008.

[9] X. Xu, "Positive solutions for singular $m$-point boundary value problems with positive parameter," Journal of Mathematical Analysis and Applications, vol. 291, no. 1, pp. 352-367, 2004.

[10] R. Ma and N. Castaneda, "Existence of solutions of nonlinear $m$-point boundary-value problems," Journal of Mathematical Analysis and Applications, vol. 256, no. 2, pp. 556-567, 2001.

[11] J. Zhao, Z. Liu, and L. Liu, "The existence of solutions of infinite boundary value problems for first-order impulsive differential systems in Banach spaces," Journal of Computational and Applied Mathematics, vol. 222, no. 2, pp. 524-530, 2008.

[12] G. Zhang and J. X. Sun, "Positive solutions of $m$-point boundary value problems," Journal of Mathematical Analysis and Applications, vol. 291, no. 2, pp. 406-418, 2004.

[13] Y.-L. Zhao and H.-B. Chen, "Existence of multiple positive solutions for $m$-point boundary value problems in Banach spaces," Journal of Computational and Applied Mathematics, vol. 215, no. 1, pp. 79 90, 2008.

[14] B. Liu, "Positive solutions of a nonlinear four-point boundary value problems in Banach spaces," Journal of Mathematical Analysis and Applications, vol. 305, no. 1, pp. 253-276, 2005.

[15] D. J. Guo, "Existence of positive solutions for $n$ th-order nonlinear impulsive singular integrodifferential equations in Banach spaces," Nonlinear Analysis: Theory, Methods E Applications, vol. 68, no. 9, pp. 2727-2740, 2008.

[16] D. J. Guo and V. Lakshmikantham, "Coupled fixed points of nonlinear operators with applications," Nonlinear Analysis: Theory, Methods E Applications, vol. 11, no. 5, pp. 623-632, 1987. 